\title{
Correction to Effects of Oligomer Length, Solvents, and Temperature on the Self- Association of Aromatic Oligoamide Foldamers
}

Yongye Zhao, ${ }^{\dagger, \$ \nabla}$ Alan L. Connor, $\$ \nabla \nabla$ Thomas A. Sobiech, ${ }^{\ddagger}$ and Bing Gong ${ }^{* \dagger, \$}$

The analytical data of oligoamides $\mathbf{5 a}$ and $\mathbf{5 b}$ presented in the Supporting Information contain wrongly entered values. The NMR and mass spectra of $\mathbf{5} \mathbf{a}$ and $\mathbf{5 b}$ were misplaced and are replaced with the correct spectra.

\section{Analytical data}

The analytical data of oligoamides $\mathbf{5 a}$ and $\mathbf{5 b}$ on pages S11 and S12 in the Supporting Information should be replaced by the following:

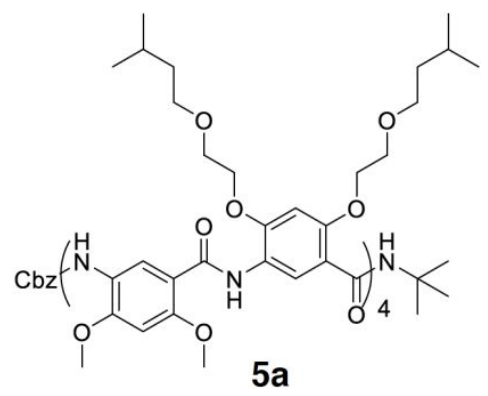

Compound 5a: ${ }^{1} \mathrm{H}$ NMR (500 MHz, $\left.\mathrm{CDCl}_{3} / \mathrm{DMSO}_{-} d_{6}(1 / 1, \mathrm{v} / \mathrm{v})\right) \delta 10.30$ (s, $\left.1 \mathrm{H}\right), 10.28(\mathrm{~s}, 1 \mathrm{H}), 10.26$ $(\mathrm{s}, 1 \mathrm{H}), 10.24(\mathrm{~s}, 1 \mathrm{H}), 10.11(\mathrm{~s}, 1 \mathrm{H}), 10.10(\mathrm{~s}, 1 \mathrm{H}), 10.05(\mathrm{~s}, 1 \mathrm{H}), 9.19(\mathrm{~s}, 2 \mathrm{H}), 9.16(\mathrm{~s}, 1 \mathrm{H}), 9.01(\mathrm{~s}$, $1 \mathrm{H}), 9.00(\mathrm{~s}, 1 \mathrm{H}), 8.90(\mathrm{~s}, 1 \mathrm{H}), 8.81(\mathrm{~s}, 1 \mathrm{H}), 8.34(\mathrm{~s}, 1 \mathrm{H}), 8.08(\mathrm{~s}, 1 \mathrm{H}), 7.32-7.16(\mathrm{~m}, 5 \mathrm{H}), 6.88(\mathrm{~m}$, $2 \mathrm{H}), 6.86(\mathrm{~s}, 1 \mathrm{H}), 6.77(\mathrm{~m}, 2 \mathrm{H}), 6.73(\mathrm{~s}, 1 \mathrm{H}), 6.66(\mathrm{~s}, 1 \mathrm{H}), 6.65(\mathrm{~s}, 1 \mathrm{H}), 5.02(\mathrm{~s}, 2 \mathrm{H}), 4.42-4.24(\mathrm{~m}$, 14H), $4.13-3.98(\mathrm{~m}, 23 \mathrm{H}), 3.88-3.77(\mathrm{~m}, 17 \mathrm{H}), 3.68(\mathrm{t}, \mathrm{J}=4.8 \mathrm{~Hz}, 2 \mathrm{H}), 3.51-3.40$ (m, 16H), 1.67 - 1.57 (m, 5H), $1.55-1.46(\mathrm{~m}, 3 \mathrm{H}), 1.42(\mathrm{~s}, 9 \mathrm{H}), 1.42-1.35(\mathrm{~m}, 10 \mathrm{H}), 1.29-1.23(\mathrm{~m}, 6 \mathrm{H}), 0.84-$ $0.78(\mathrm{~m}, 30 \mathrm{H}), 0.74-0.70(\mathrm{~m}, 18 \mathrm{H}) .{ }^{13} \mathrm{C} \mathrm{NMR}\left(100 \mathrm{MHz}, \mathrm{CDCl}_{3} / \mathrm{DMSO}-d_{6}(1 / 1, \mathrm{v} / \mathrm{v})\right) \delta 165.45$, $162.51,162.37,162.32,162.13,161.87,155.00,154.83,154.64,153.71,153.52,153.43,153.26$, 153.12 , 151.58, 151.08, 136.91, 128.50, 128.04, 122.93, 122.11, 121.93, 114.21, 114.07, 113.46, $113.35,113.21,80.09,69.53,69.48,69.44,69.39,69.34,68.95,68.89,68.78,66.17,56.76,56.66$, $56.52,56.29,38.56,38.45,38.39,28.25,24.99,24.96,24.88,22.81,22.77,22.74,22.68$. MALDI (m/z) calcd. for $\mathrm{C}_{132} \mathrm{H}_{184} \mathrm{~N}_{8} \mathrm{O}_{35} \mathrm{Na} 2465.2790\left(\mathrm{M}+\mathrm{Na}^{+}\right)$, found 2465.3107. 


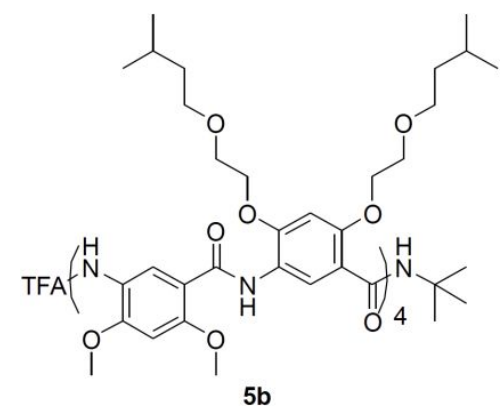

Compound 5b: ${ }^{1} \mathrm{H}$ NMR $\left(500 \mathrm{MHz}, \mathrm{CDCl}_{3} / \mathrm{DMSO}_{-} d_{6}(1 / 1, \mathrm{v} / \mathrm{v})\right) \delta 10.37$ (s, 1H), $10.29(\mathrm{~s}, 3 \mathrm{H}), 10.23$ (s, 1H), 10.12 (s, 2H), $10.05(\mathrm{~s}, 1 \mathrm{H}), 9.21(\mathrm{~s}, 2 \mathrm{H}), 9.18(\mathrm{~s}, 1 \mathrm{H}), 9.03(\mathrm{~s}, 2 \mathrm{H}), 8.90(\mathrm{~s}, 1 \mathrm{H}), 8.85(\mathrm{~s}, 1 \mathrm{H})$, $8.10(\mathrm{~s}, 1 \mathrm{H}), 6.92(\mathrm{~s}, 2 \mathrm{H}), 6.91(\mathrm{~s}, 1 \mathrm{H}), 6.81(\mathrm{~s}, 2 \mathrm{H}), 6.78(\mathrm{~s}, 2 \mathrm{H}), 6.72(\mathrm{~s}, 1 \mathrm{H}), 4.44(\mathrm{~m}, 6 \mathrm{H}), 4.39(\mathrm{~m}$, $6 \mathrm{H}), 4.31(\mathrm{~m}, 2 \mathrm{H}), 4.17-4.10(\mathrm{~m}, 14 \mathrm{H}), 4.05(\mathrm{~s}, 9 \mathrm{H}), 3.93(\mathrm{~s}, 3 \mathrm{H}), 3.90-3.81(\mathrm{~m}, 14 \mathrm{H}), 3.72(\mathrm{~m}$, 2H), $3.54-3.45(\mathrm{~m}, 16 \mathrm{H}), 1.73-1.60(\mathrm{~m}, 4 \mathrm{H}), 1.61-1.50(\mathrm{~m}, 4 \mathrm{H}), 1.46(\mathrm{~s}, 9 \mathrm{H}), 1.45-1.39(\mathrm{~m}$, 10H), $1.30(\mathrm{~m}, 6 \mathrm{H}), 0.94-0.70(\mathrm{~m}, 48 \mathrm{H}) .{ }^{13} \mathrm{C} \mathrm{NMR}\left(125 \mathrm{MHz}, \mathrm{CDCl}_{3} / \mathrm{DMSO}_{6}(1 / 1, \mathrm{v} / \mathrm{v})\right) \delta 165.56$, $162.18,157.78,157.22,153.52,153.21,151.59,122.96,122.16,114.19,113.57,113.43,113.23,80.14$, 69.67, 69.40, 69.34, 68.95, 56.83, 56.59, 38.57, 38.46, 28.25, 25.01, 24.98, 24.95, 24.90, 22.78, 22.70. Maldi (m/z) calcd. for $\mathrm{C}_{126} \mathrm{H}_{177} \mathrm{~N}_{8} \mathrm{~F}_{3} \mathrm{O}_{34} \mathrm{Na} 2427.2245\left(\mathrm{M}+\mathrm{Na}^{+}\right)$, found 2427.2510. 


\section{NMR and mass spectra}

The ${ }^{1} \mathrm{H}$ NMR, ${ }^{13} \mathrm{C}$ NMR and mass spectra of 5a and $\mathbf{5 b}$ on page S23 - S26 in the Supporting Information should be replaced with the following spectra:
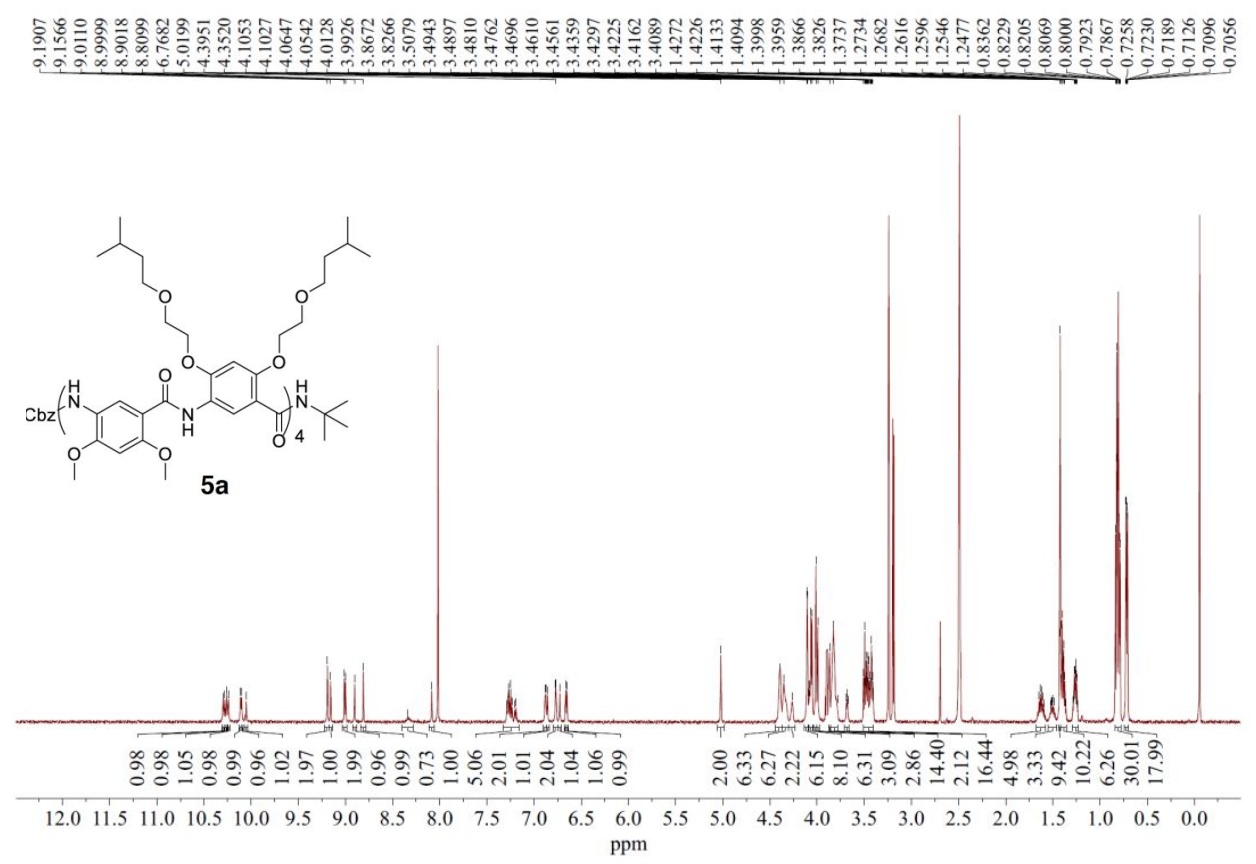

${ }^{1} \mathrm{H}$ NMR spectrum of $5 \mathbf{a}\left(500 \mathrm{MHz}, \mathrm{CDCl}_{3} / \mathrm{DMSO}_{-}(1 / 1, \mathrm{v} / \mathrm{v})\right)$
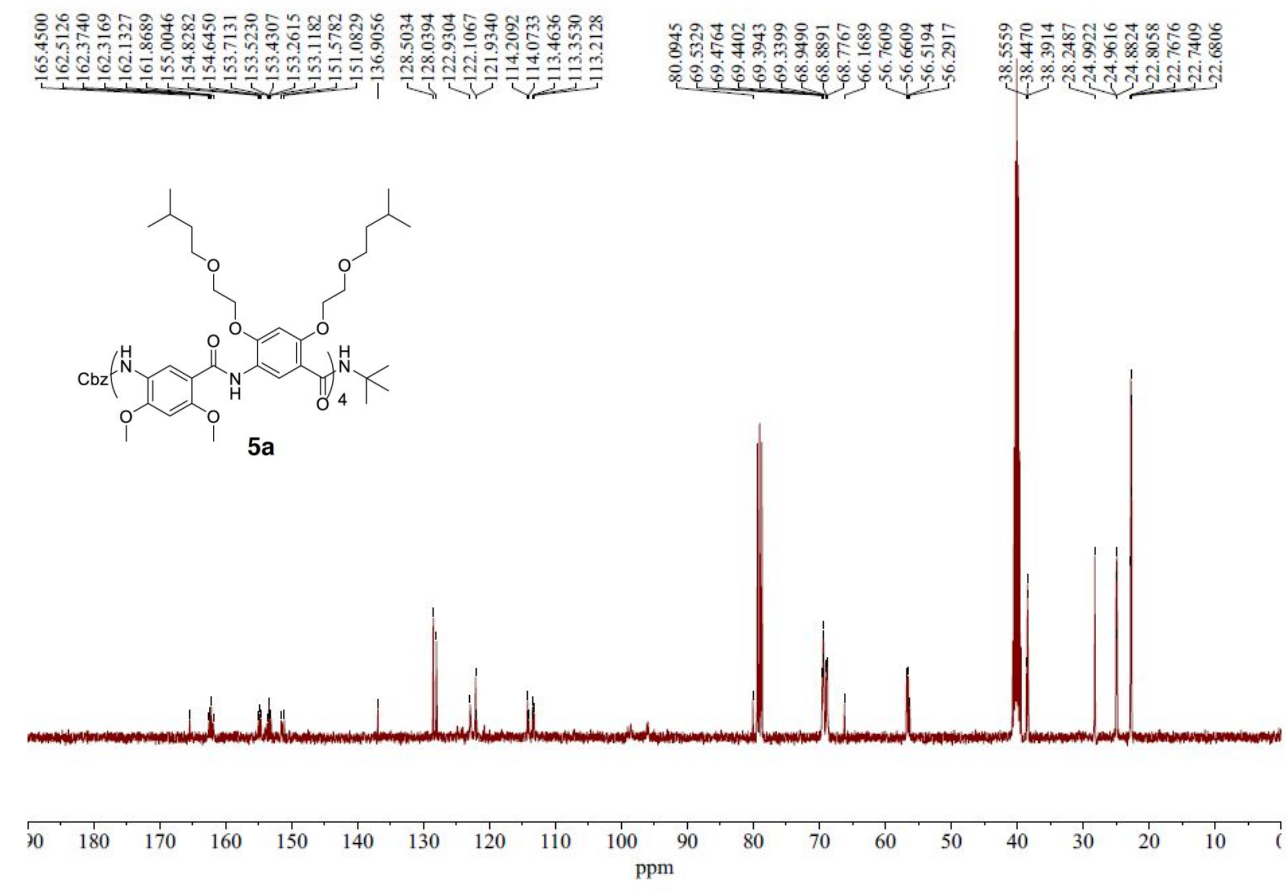

${ }^{13} \mathrm{C}$ NMR spectrum of $5 \mathbf{a}\left(100 \mathrm{MHz}, \mathrm{CDCl}_{3} / \mathrm{DMSO}_{-} d_{6}(1 / 1, \mathrm{v} / \mathrm{v})\right)$ 


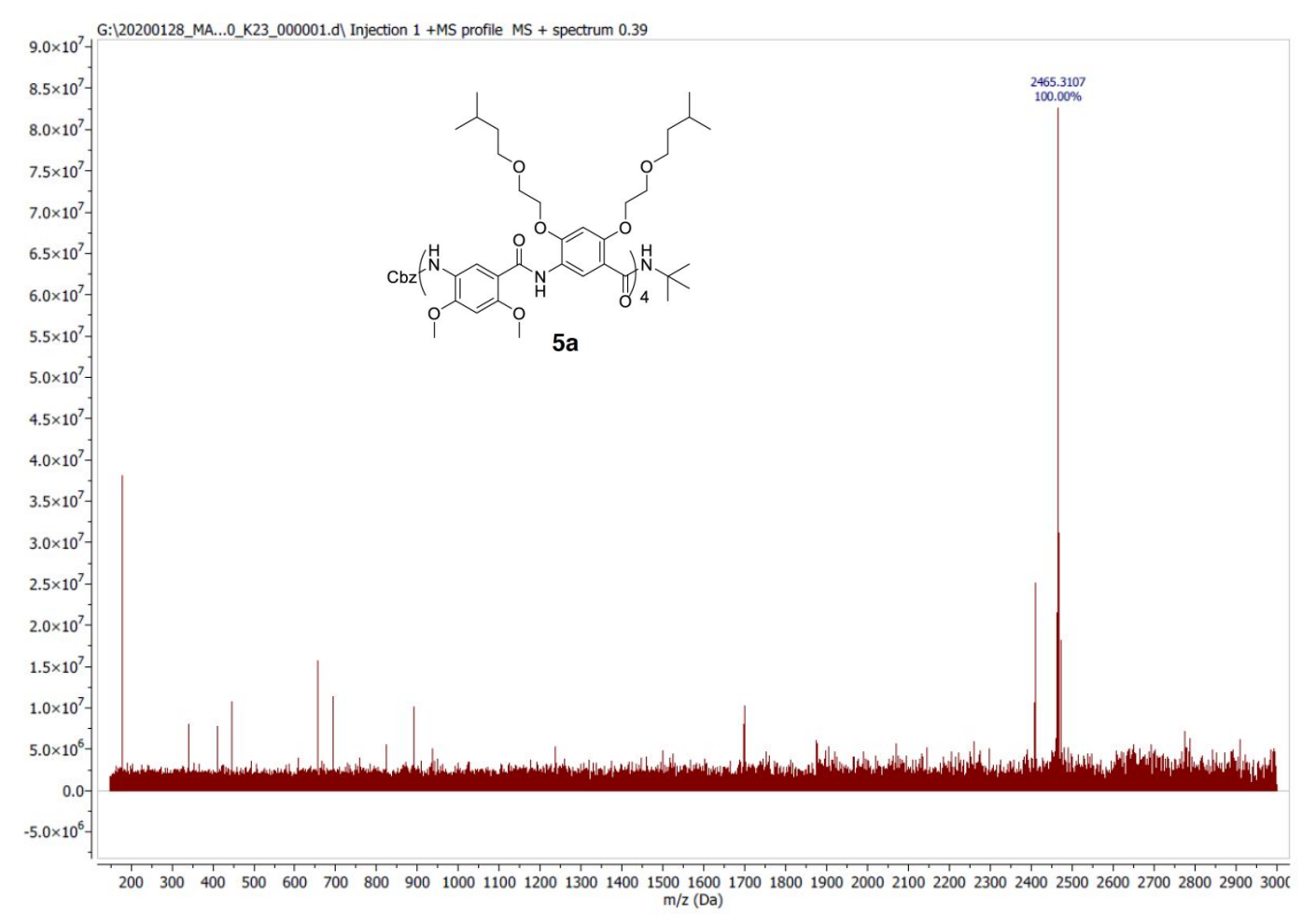

Mass (MALDI) spectrum of $\mathbf{5 a}$

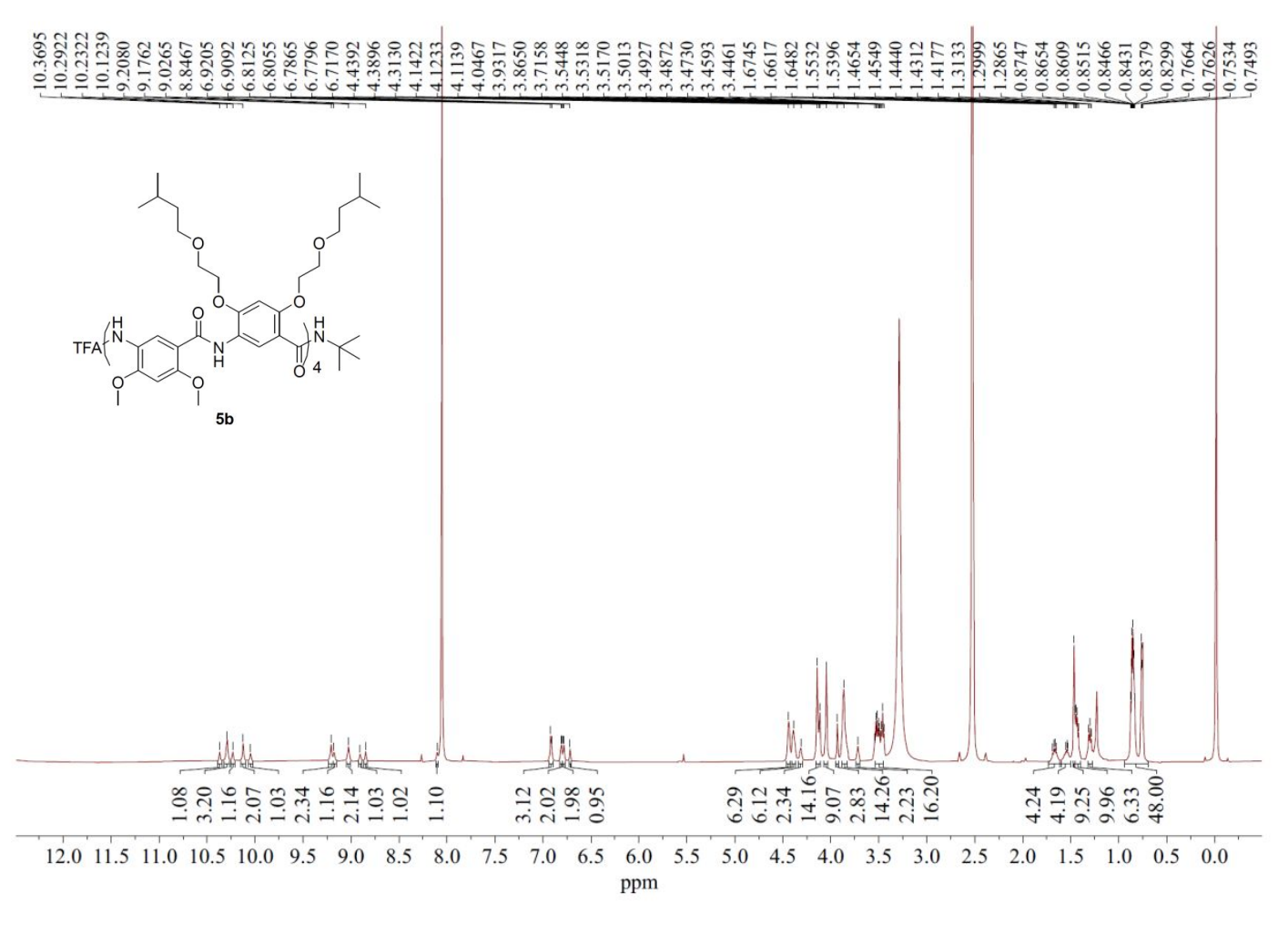

${ }^{1} \mathrm{H}$ NMR spectrum of $\mathbf{5 b}\left(500 \mathrm{MHz}, \mathrm{CDCl}_{3} / \mathrm{DMSO}_{-} d_{6}(1 / 1, \mathrm{v} / \mathrm{v})\right)$ 

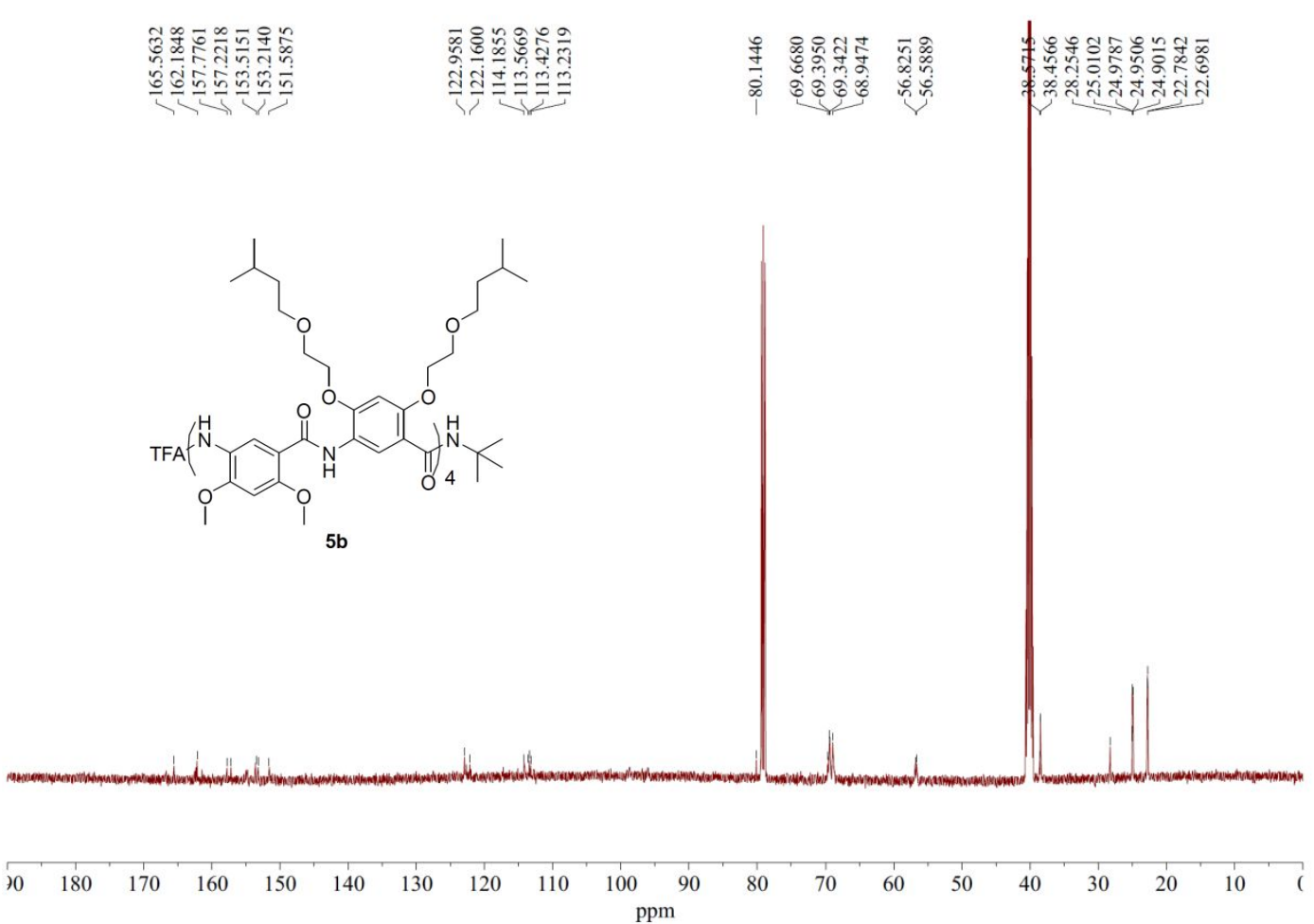

${ }^{1} \mathrm{H}$ NMR spectrum of $\mathbf{5 b}\left(125 \mathrm{MHz}, \mathrm{CDCl}_{3} / \mathrm{DMSO}_{-}(1 / 1, \mathrm{v} / \mathrm{v})\right)$

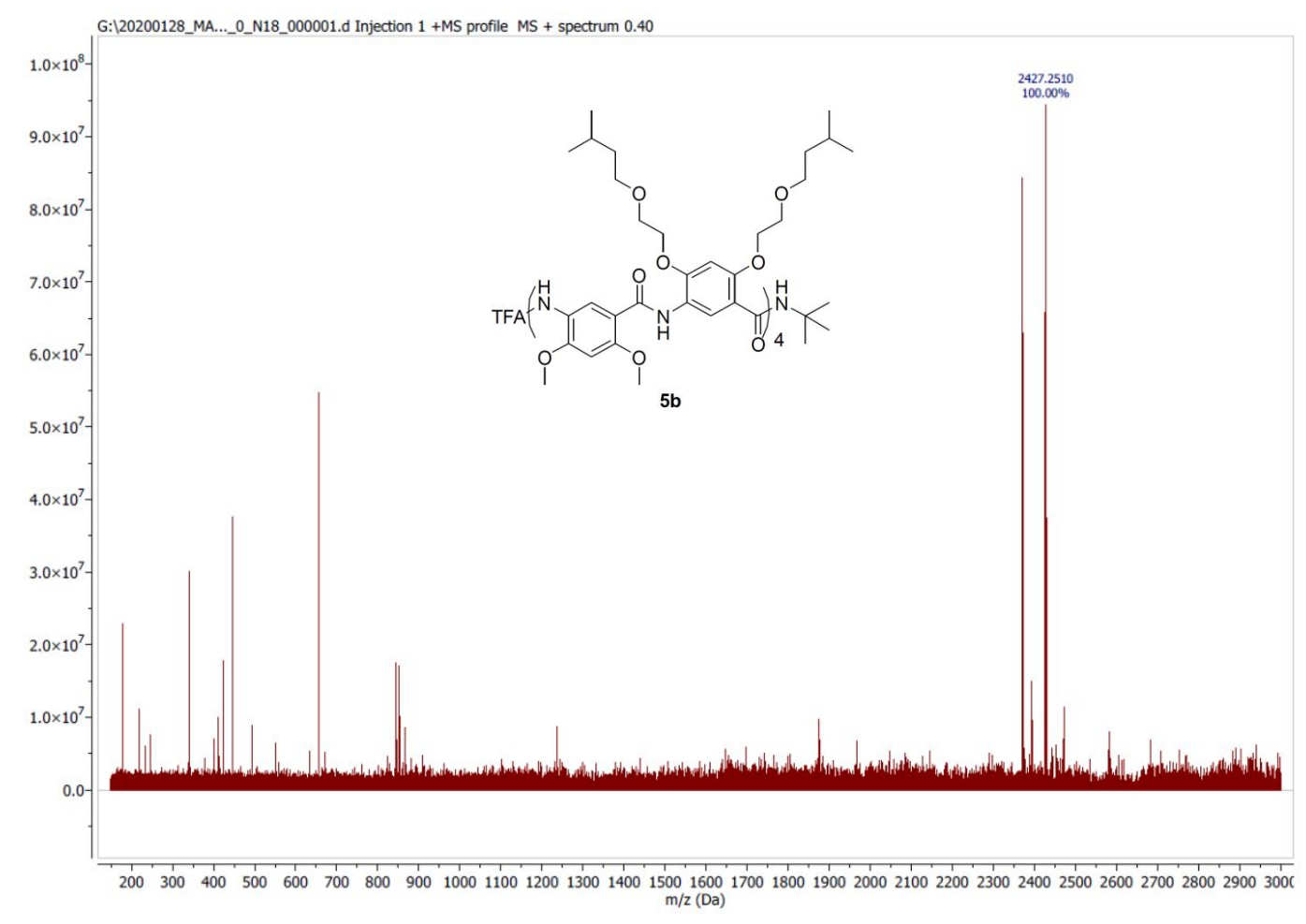

Mass (MALDI) spectrum of $\mathbf{5 b}$ 